Article

\title{
Eco-Friendly Synthesis, Characterization and Biological Evaluation of Some Novel Pyrazolines Containing Thiazole Moiety as Potential Anticancer and Antimicrobial Agents
}

\author{
Mastoura M. Edrees ${ }^{1,2}, *\left(\mathbb{D}\right.$, Sraa Abu-Melha ${ }^{1}$, Amirah M. Saad ${ }^{3}$, Nabila A. Kheder ${ }^{3,4}$, \\ Sobhi M. Gomha ${ }^{4, *(1)}$ and Zeinab A. Muhammad ${ }^{2}$ \\ 1 Department of Chemistry, Faculty of Science, King Khalid University, Abha 61413, Saudi Arabia; \\ sabomlba@kku.edu.sa \\ 2 Department of Organic Chemistry, National Organization for Drug Control and Research (NODAR), \\ Giza 12311, Egypt; zeinab.a.muhammad@gmail.com \\ 3 Department of Pharmaceutical Chemistry, Faculty of Pharmacy, King Khalid University, \\ Abha 61441, Saudi Arabia; ammaalqahtani@kku.edu.sa (A.M.S.); nabila.abdelshafy@gmail.com (N.A.K.) \\ 4 Department of chemistry, Faculty of Science, Cairo University, Giza 12613, Egypt \\ * Correspondence: mmohamededrees@yahoo.com or mstorh@kku.edu.sa (M.M.E.); \\ s.m.gomha@gmail.com (S.M.G.); Tel.: +966-545-888-764 (M.M.E.); +20-237-400-304 (S.M.G.); \\ Fax: +2-025-685-799 (S.M.G.)
}

Academic Editors: Philippe Belmont and Richard A. Bunce

Received: 11 October 2018; Accepted: 12 November 2018; Published: 14 November 2018

\begin{abstract}
The one-pot synthesis of a series of pyrazoline derivatives containing the bioactive thiazole ring has been performed through a 1,3-dipolar cycloaddition reaction of $\mathrm{N}$-thiocarbamoylpyrazoline and different hydrazonoyl halides or $\alpha$-haloketones in the presence of DABCO (1,4-diazabicyclo[2.2.2] octane) as an eco-friendly catalyst using the solvent-drop grinding method. The structure of the synthesized compounds was elucidated using elemental and spectroscopic analyses (IR, NMR, and Mass). The activity of these compounds against human hepatocellular carcinoma cell line (HepG2) was tested and the results showed that the pyrazoline 11f, which has a fluorine substituent, is the most active. The antimicrobial activities of the newly synthesized compounds were determined against two fungi and four bacterial strains, and the results indicated that some of the newly synthesized pyrazolines are more potent than the standard drugs against test organisms.
\end{abstract}

Keywords: pyrazolines; thiazoles; hydrazonoyl halides; antimicrobial activity; anticancer activity

\section{Introduction}

The majority of antitumor drugs have many serious side effects. Another fundamental problem in chemotherapy is the emergence of cancer cell drug resistance [1]. Also, there is a rapid evolution of drug-resistant pathogens that raise the need for the discovery of new drugs to counterbalance the effects of this resistance. Pyrazolines have been found to possess diverse biological activities such as anticancer [2-4], antitumor [5], antioxidant [6], antimicrobial [7,8], antitubercular [9], antimalarial [10], anti-amoebic [11], DPPH radical scavenging, anti-diabetic [12], antiviral [13] and amine oxidase [14]. Also, thiazoles are known to have anticonvulsant [15], antimicrobial [16], anti-inflammatory [17], anticancer [18-20], antidiabetic [21], anti-HIV [22], anti-Alzheimer [23], antihypertensive [24], antifungal [25], and antioxidant [26] activities. In view of the above mentioned findings and as a part of our research interest towards developing new ways to synthesize a variety of heterocyclic systems with promising biological and pharmacological activities [27-30], we present in this research an efficient 
synthesis of a series of pyrazolines attached to thiazole moiety using $N$-thiocarbamoylpyrazoline [31] and the appropriate hydrazonoyl halides [32-34] or $\alpha$-haloketones in the presence of DABCO under the solvent-drop grinding method [35].

\section{Results and Discussion}

\subsection{Chemistry}

$N$-Thiocarbamoylpyrazoline 2 was prepared by cyclization of 3-(2,4-dichlorophenyl)-1-(thiophen2-yl)prop-2-en-1-one (1) [31] with thiosemicarbazide in the presence of DABCO as a basic catalyst using a grinding method at room temperature as shown in Scheme 1. The reaction between the pyrazoline $\mathbf{2}$ and the appropriate hydrazonoyl halides 3a-f [32-34] in the presence of DABCO using the solvent-drop grinding method afforded a series of pyrazolines attached to the bioactive thiazole moiety (Scheme 1). The structures of the synthesized compounds $\mathbf{5 a}-\mathbf{f}$ were elucidated using elemental and spectroscopic analysis (IR, NMR, and Mass). The ${ }^{1} \mathrm{H}-\mathrm{NMR}$ of pyrazolines $\mathbf{5 a}-\mathbf{f}$ showed in each case a singlet signal at $\delta 2.16-2.49 \mathrm{ppm}$ due to the methyl protons, in addition to the expected protons of pyrazoline and aromatic rings. Also, their mass spectra showed in each case a molecular ion peak which agrees with the proposed structures (see the Experimental section). The reaction was assumed to start intially through nucleophilic displacement of the halide to afford intermediate 4, which underwent intramolecular cyclization and dehydration to afford the final product 5 (Scheme 1).

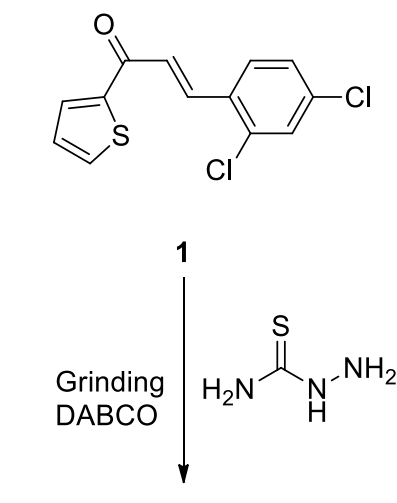<smiles>NC(=S)N1N=C(c2cccs2)CC1c1ccc(Cl)cc1Cl</smiles>

2<smiles>Cc1nc(N2N=C(c3cccs3)CC2c2ccc(Cl)cc2Cl)sc1N=[W]</smiles>

$5 a-f$<smiles>CC(=O)/C(=N\N[Al])SC(=N)N1N=C(c2cccs2)CC1c1ccc(Cl)cc1Cl</smiles>

4a-f

3-6: Ar: a, $\mathrm{C}_{6} \mathrm{H}_{5} ; \mathrm{b}, 4-\mathrm{CH}_{3} \mathrm{C}_{6} \mathrm{H}_{4} ; \mathrm{c}, 4-\mathrm{CH}_{3} \mathrm{OC}_{6} \mathrm{H}_{4} ; \mathrm{d}, 4-\mathrm{ClC}_{6} \mathrm{H}_{4} ; \mathrm{e}, 4-\mathrm{BrC}_{6} \mathrm{H}_{4} ; \mathrm{f}, 4-\mathrm{NO}_{2} \mathrm{C}_{6} \mathrm{H}_{4}$

Scheme 1. Synthesis of pyrazoline derivatives $\mathbf{5 a}-\mathbf{f}$.

Next, the pyrazoline derivative $\mathbf{2}$ was reacted with the appropriate hydrazonoyl halides $\mathbf{6 a}-\mathbf{e}$ [36] under the same experimental conditions to give the pyrazolylthiazolone derivatives 8a-e (Scheme 2). The IR spectra of pyrazolines 8a-e revealed in each case absorption bands at $v=1649-1686$, and $3430-3434 \mathrm{~cm}^{-1}$ corresponding to carbonyl and $\mathrm{NH}$-hydrazo groups, respectively. Also, their ${ }^{1} \mathrm{H}-\mathrm{NMR}$ spectra revealed a singlet signal in the region $\delta 11.17-11.74 \mathrm{ppm}$ due to the NH proton. In addition, their mass spectra showed the expected peaks due to their molecular ions. 

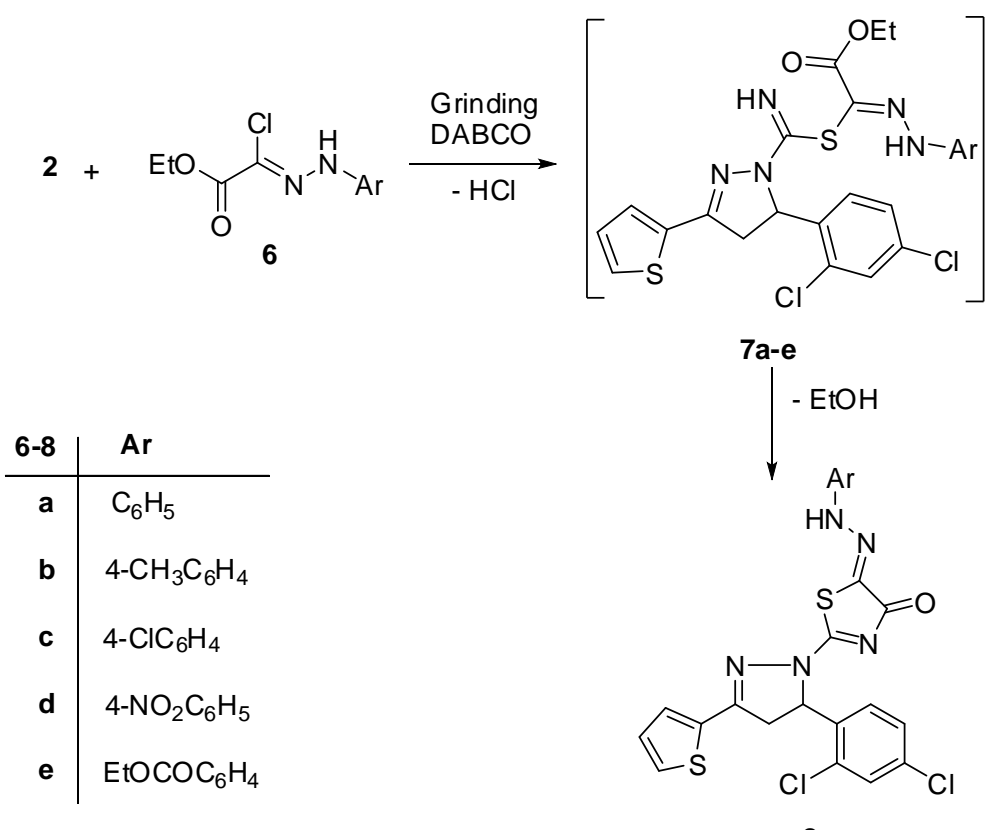

$8 a-e$

Scheme 2. Synthesis of pyrazolylthiazolons $8 \mathbf{a}-\mathbf{e}$.

The reaction was suggested to proceed through nucleophilic displacement of chloride to give intermediate 7 which underwent elimination of one ethanol molecule to afford the target products 8a-e.

Finally, the reaction between the pyrazoline derivative 2 and appropriate $\alpha$-haloketones $\mathbf{9 a - h}$ afforded pyrazolylthiazole derivatives 11a-h (Scheme 3). The proposed structures are consistent with the analytical and spectroscopic analyses (see the Experimental section).

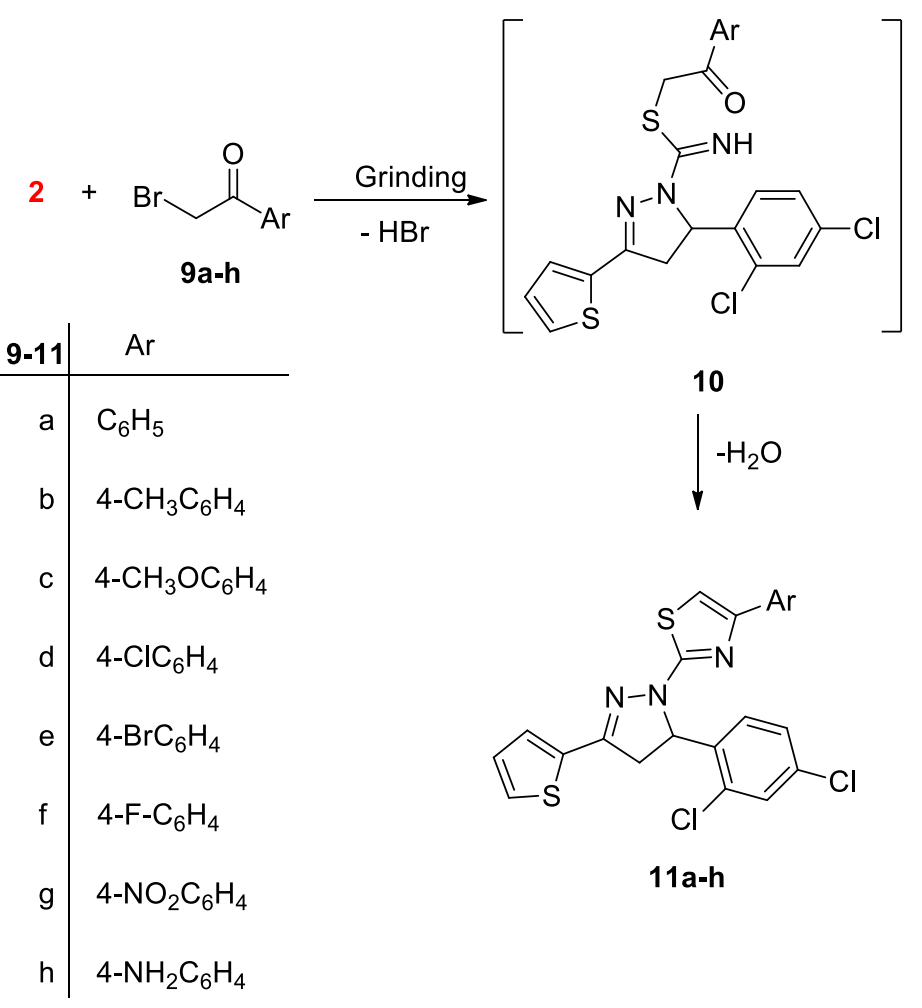

Scheme 3. Synthesis of pyrazolylthiazoles 11a-h. 


\subsection{Pharmacology}

\subsubsection{Antitumor Activity}

The antitumor activity of the products $\mathbf{5 a}-\mathbf{e}$ and $\mathbf{8 a}-\mathbf{d}$, and $\mathbf{1 1 a}-\mathbf{f}$ was investigated against human hepatocellular carcinoma cell line (HepG2), in comparison with Cisplatin as the anticancer standard drug $[37,38]$. $\mathrm{IC}_{50}$ (the concentration of test compounds required to kill $50 \%$ of cell population) was determined from the dose response curve (Table 1).

Table 1. The in vitro inhibitory activity of the synthesized pyrazolines against HepG2 cell line expressed as $\mathrm{IC}_{50}$ values $(\mu \mathrm{M}) \pm$ standard deviation from three replicates.

\begin{tabular}{cc}
\hline Tested Compounds & IC $_{\mathbf{5 0}}(\boldsymbol{\mu M})$ \\
\hline $\mathbf{5 a}$ & $9.88 \pm 1.8$ \\
$\mathbf{5 b}$ & $22.40 \pm 1.1$ \\
$\mathbf{5 c}$ & $20.10 \pm 2.7$ \\
$\mathbf{5 d}$ & $5.78 \pm 1.8$ \\
$\mathbf{5 e}$ & $12.4 \pm 2.9$ \\
$\mathbf{8 a}$ & $8.44 \pm 1.9$ \\
$\mathbf{8 b}$ & $13.90 \pm 2.1$ \\
$\mathbf{8 c}$ & $3.54 \pm 1.8$ \\
$\mathbf{8 d}$ & $7.68 \pm 1.9$ \\
$\mathbf{1 1 a}$ & $8.12 \pm 1.2$ \\
$\mathbf{1 1 b}$ & $14.91 \pm 1.8$ \\
$\mathbf{1 1 d}$ & $2.98 \pm 1.8$ \\
$\mathbf{1 1 f}$ & $1.70 \pm 8.2$ \\
Cisplatin & $0.90 \pm 1.1$ \\
\hline
\end{tabular}

The data are expressed in the form of mean \pm standard error.

Figure 1 shows a comparison between the values of $\mathrm{IC}_{50}$ of the evaluated pyrazolines against Cisplatin, which is used as a standard drug.

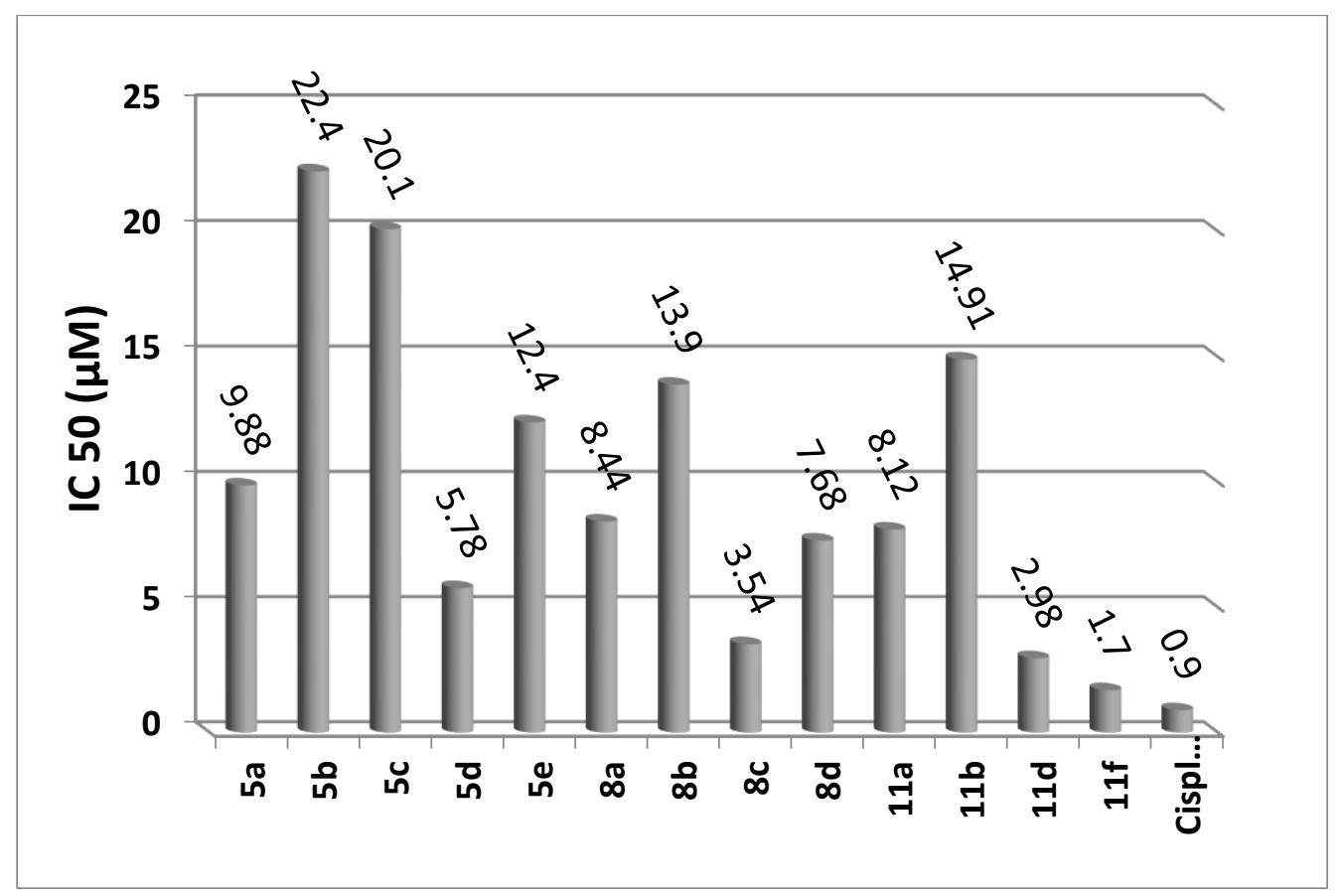

Figure 1. Cytotoxic activities of tested compounds against HepG2. 
The $\mathrm{IC}_{50}$ of cytotoxic activity data (Table 1 and Figure 1 ) showed that the pyrazoline derivative 11f, which bears a fluorine substituent had the highest cytotoxic activity compared to Cisplatin.

From the obtained results, we suggest the following structural requirements for activity:

- The phenyl ring substitution $(\mathrm{X})$ is important for activity.

- The electronic nature of phenyl ring substitution $(X)$ is important.

- Compounds with an electron withdrawing para-substituents demonstrate good activity.

- A phenyl ring substituted at the C-4 position by halogens has increased activity.

- A fluorine atom at the para position of the phenyl ring optimizes activity.

- Phenyl rings with para alkyl groups show decreased activity.

\subsubsection{Evaluation of the Antimicrobial Activity}

The in vitro antimicrobial activities of the newly synthesized compounds and reference drugs were tested by inhibition zone technique $[39,40]$ and minimum inhibitory concentration (MIC), using two fungi: Aspergillus fumigatus (RCMB 002008 (4) and Candida albicans (RCMB 05036), two gram-positive bacteria: Staphylococcus aureus (RCMB 010010), and Bacillus subtilis (RCMB 010067), two gram-negative bacteria: Escherichia coli (RCMB 010052), and Proteus vulgaris RCMB 004 (1) ATCC 13315, and the results are depicted in Tables 2 and 3. The data showed that some of the newly synthesized pyrazolines are able to inhibit the growth of the examined microbes in vitro and some of them are more potent than the standard drugs. In general, the chemical structure of the whole molecule, comprising the nature of the heterocyclic system as well as the type of the substituted function present in the heterocyclic ring structure, has a pronounced effect. All data were recorded as the mean of three replicates with standard deviation $( \pm \mathrm{SD})$ using the software Excel (Microsoft, New York, NY, USA).

From the screening results, it can be seen that:

- Pyrazoline derivatives $\mathbf{1 1}$ have higher activity than $\mathbf{5}$ and $\mathbf{8}$ against the tested bacteria and fungi

- Pyrazoline derivatives 11a, 11b, 11d, and 11e are the most potent compounds against Aspergillus fumigatus and they had higher potency than the standard drug, Ketoconazole.

- The pyrazoline 11a exhibited high antifungal activity against $C A$, and is more potent than the standard drug Ketoconazole.

- The pyrazoline derivative 11a is more potent than the reference drug Gentamycin against $S A$ and $P V$.

- The higher antimicrobial activity of pyrazolyl-thiazole derivative 11a is due to the phenyl group at position 4 of the thiazole ring.

- Most of the tested compounds have higher activity against gram positive bacteria than gram negative bacteria.

- Compound 5e gave no action with all the tested species

- Most of the tested compounds have higher activity against bacteria than fungi

- Pyrazoline derivatives $\mathbf{8}$ have higher activity against $P V$ than $E C$

In addition, the minimum inhibitory concentration (MIC) of compounds $\mathbf{5 a}-\mathbf{f}, \mathbf{8} \mathbf{a}-\mathbf{e}$ and $\mathbf{1 1 a}-\mathbf{h}$ was considered to be the lowest concentration of the tested substance exhibiting no visible growth of the bacteria or fungi on the plate as shown in Table 3.

The results of minimum inhibitory concentration of the tested compounds $5 \mathbf{a}-\mathbf{f}, \mathbf{8 a}-\mathbf{e}$ and $11 \mathbf{a}-\mathbf{h}$ exhibited that:

- The synthesized pyrazolines showed a broad spectrum of activities with MIC values from 9.77-10,000 $\mu \mathrm{g} \mathrm{mL}^{-1}$.

- Compound 11a is the most active compound against all the tested microorganisms. 
Table 2. Antimicrobial activities of the new pyrazolines 5a-f, 8a-e and 11a-h expressed as inhibition diameter zones in millimeters ( $\mathrm{mm}$ ) based on well diffusion assay.

\begin{tabular}{|c|c|c|c|c|c|c|}
\hline \multirow{3}{*}{ Sample } & \multicolumn{6}{|c|}{ Microorganisms } \\
\hline & \multicolumn{2}{|c|}{ FUNGI } & \multicolumn{2}{|c|}{ Gram Positive Bacteria } & \multicolumn{2}{|c|}{ Gram Negative Bacteria } \\
\hline & $A F$ & $C A$ & $S A$ & $B S$ & $E C$ & $P V$ \\
\hline $5 a$ & NA & $9 \pm 0.4$ & $10 \pm 0.7$ & NA & $8 \pm 0.2$ & $10 \pm 0.7$ \\
\hline $5 b$ & NA & NA & $10 \pm 0.80$ & $9 \pm 0.4$ & $11 \pm 0.6$ & $13 \pm 0.6$ \\
\hline $5 c$ & $10 \pm 0.4$ & NA & $13 \pm 0.7$ & $12 \pm 0.4$ & $11 \pm 0.5$ & $10 \pm 0.7$ \\
\hline $5 d$ & NA & NA & $9 \pm 0.2$ & $10 \pm 0.3$ & $9 \pm 0.4$ & $12 \pm 0.9$ \\
\hline $5 e$ & NA & NA & NA & NA & NA & NA \\
\hline $5 f$ & NA & NA & $12 \pm 0.6$ & $13 \pm 0.5$ & $12 \pm 0.9$ & $15 \pm 1.1$ \\
\hline $8 a$ & NA & NA & $11 \pm 0.7$ & $13 \pm 0.7$ & $11 \pm 0.8$ & $15 \pm 0.9$ \\
\hline $8 b$ & NA & NA & $9 \pm 0.3$ & $12 \pm 0.8$ & $13 \pm 0.9$ & $12 \pm 0.6$ \\
\hline $8 c$ & NA & NA & $10 \pm 0.3$ & $13 \pm 0.4$ & $15 \pm 0.7$ & $14 \pm 0.4$ \\
\hline $8 d$ & NA & NA & $11 \pm 0.6$ & $13 \pm 0.7$ & $12 \pm 0.4$ & $15 \pm 0.5$ \\
\hline $8 e$ & NA & NA & $12 \pm 0.7$ & $11 \pm 0.4$ & $13 \pm 0.6$ & $14 \pm 0.8$ \\
\hline $11 a$ & $28 \pm 0.9$ & $23 \pm 1.1$ & $25 \pm 1.4$ & $18 \pm 0.6$ & $17 \pm 0.6$ & $28 \pm 1.4$ \\
\hline $11 b$ & $21 \pm 0.8$ & $14 \pm 0.6$ & $12 \pm 0.5$ & $11 \pm 0.6$ & $12 \pm 0.6$ & $14 \pm 0.7$ \\
\hline 11c & $12 \pm 0.8$ & $10 \pm 0.4$ & NA & $12 \pm 0.7$ & $13 \pm 0.9$ & $11 \pm 0.6$ \\
\hline $11 d$ & $20 \pm 1.2$ & NA & $10 \pm 0.7$ & $9 \pm 0.4$ & $10 \pm 0.4$ & $12 \pm 0.3$ \\
\hline $11 \mathrm{e}$ & $25 \pm 0.7$ & NA & $9 \pm 0.5$ & NA & NA & $10 \pm 0.3$ \\
\hline $11 \mathrm{f}$ & NA & NA & $10 \pm 0.4$ & $11 \pm 0.6$ & $12 \pm 0.9$ & $11 \pm 0.3$ \\
\hline $11 \mathrm{~g}$ & NA & $12 \pm 0.7$ & $11 \pm 0.5$ & $14 \pm 0.6$ & $15 \pm 0.7$ & $16 \pm 0.9$ \\
\hline $11 \mathrm{~h}$ & NA & NA & $12 \pm 0.9$ & $13 \pm 0.4$ & $14 \pm 0.8$ & $13 \pm 0.5$ \\
\hline Ketoconazole & $17 \pm 0.4$ & $20 \pm 0.8$ & - & - & - & - \\
\hline Gentamycin & - & - & $24 \pm 1.2$ & $26 \pm 0.7$ & $30 \pm 0.9$ & $25 \pm 0.8$ \\
\hline
\end{tabular}

NA. No activity, data are expressed in the form of mean of inhibition zone diameter (mm) for test compounds and performed in triplicate \pm SD; AF (Aspergillus fumigatus (RCMB 002008 (4)), CA (Candida albicans (RCMB 05036), SA (Staphylococcus aureus CMB 010010)), BS (Bacillus subtilis (RCMB 010067)), EC (Escherichia coli (RCMB 010052)), PV (Proteus vulgaris RCMB 004 (1) ATCC 13315).

Table 3. Antimicrobial activities of the newly synthesized pyrazolines $\mathbf{5 a}-\mathbf{f}, \mathbf{8} \mathbf{a}-\mathbf{e}$ and $\mathbf{1 1 a}-\mathbf{h}$ were expressed as MIC in $\mu \mathrm{g} / \mathrm{mL}$.

\begin{tabular}{|c|c|c|c|c|c|c|}
\hline \multirow{3}{*}{ Sample } & \multicolumn{6}{|c|}{ Microorganisms } \\
\hline & \multicolumn{2}{|c|}{ FUNGI } & \multicolumn{2}{|c|}{ Gram Positive Bacteria } & \multicolumn{2}{|c|}{ Gram Negative Bacteria } \\
\hline & $A F$ & $C A$ & $S A$ & $B S$ & $E C$ & $P V$ \\
\hline $5 a$ & - & 10,000 & 5000 & - & 10,000 & 5000 \\
\hline $5 b$ & - & - & 10,000 & 10,000 & 5000 & 625 \\
\hline $5 c$ & 10,000 & - & 625 & 1250 & 2500 & 5000 \\
\hline $5 d$ & - & - & 5000 & 2500 & 5000 & 312.5 \\
\hline $5 e$ & - & - & - & - & - & - \\
\hline $5 f$ & - & - & 625 & 312.5 & 625 & 156.25 \\
\hline $8 a$ & - & - & 625 & 156.25 & 1250 & 39.06 \\
\hline $8 b$ & - & - & 5000 & 312.5 & 625 & 625 \\
\hline $8 c$ & - & - & 2500 & 625 & 156.25 & 312.5 \\
\hline $8 d$ & - & - & 2500 & 156.25 & 312.5 & 156.25 \\
\hline $8 e$ & - & - & 312.5 & 2500 & 78.13 & 156.25 \\
\hline $11 a$ & 312.5 & 625 & 9.77 & 78.13 & 78.13 & 19.53 \\
\hline $11 b$ & 156.25 & 1250 & 2500 & 1250 & 1250 & 312.5 \\
\hline 11c & 2500 & - & - & 1250 & 625 & 2500 \\
\hline 11d & 625 & - & 50,000 & 10,000 & 10,000 & 1250 \\
\hline $11 e$ & 312.5 & - & 5000 & - & - & 5000 \\
\hline $11 f$ & - & - & 5000 & 2500 & 625 & 2500 \\
\hline $11 \mathrm{~g}$ & - & - & 5000 & 156.25 & 78.13 & 78.13 \\
\hline $11 \mathrm{~h}$ & - & - & 2500 & 625 & 312.5 & 625 \\
\hline
\end{tabular}




\section{Experimental}

\subsection{Chemistry}

Melting points were measured on an Electrothermal IA 9000 series digital melting point apparatus (Bibby Sci. Lim. Stone, Staffordshire, UK). IR spectra were recorded in potassium bromide discs on PyeUnicam SP 3300 and Shimadzu FTIR 8101 PC infrared spectrophotometers (Shimadzu, Tokyo, Japan). ${ }^{1} \mathrm{H}-\mathrm{NMR}$ spectra were recorded on a Varian Mercury VX-300 NMR spectrometer (Varian, Inc., Karlsruhe, Germany) operating at $300 \mathrm{MHz}\left({ }^{1} \mathrm{H}-\mathrm{NMR}\right)$ and run in deuterated dimethylsulfoxide $\left(\mathrm{DMSO}-d_{6}\right)$. Chemical shifts were related to that of the solvent. Mass spectra were recorded on a Shimadzu GCMS-QP1000 EX mass spectrometer (Tokyo, Japan) at $70 \mathrm{eV}$. Elemental analyses were measured by using a German made Elementarvario LIII CHNS analyzer. The biological evaluations of the products were carried out in the medical mycology laboratory of the regional center for mycology and biotechnology of Al-Azhar University, Cairo, Egypt. The 3-(2,4-dichlorophenyl)-1-(thiophen-2-yl)prop-2-en-1-one (1) [31], the hydrazonoyl halides $\mathbf{3 a}[32,33], \mathbf{3 b}-\mathbf{f}[34]$ and $\mathbf{6 a}-\mathbf{e}[36]$ were prepared as described in the literature.

Synthesis of 5-(2,4-dichlorophenyl)-3-(thiophen-2-yl)-4,5-dihydro-1H-pyrazole-1-carbothioamide (2). A mixture of 3-(2,4-dichlorophenyl)-1-(thiophen-2-yl)prop-2-en-1-one (1) (2.83 g, $10 \mathrm{mmol})$ and thiosemicarbazide $(0.91 \mathrm{~g}, 10 \mathrm{mmol})$ was taken in a mortar at room temperature. A catalytic amount of DABCO was added. The reaction mixture was ground by the pestle, under the hood, for $15 \mathrm{~min}$. The reaction mixture was then poured into water, and the solid product was collected by filtration. The crude product was recrystallized from ethanol as yellow crystals, $62 \%$ yield, m.p. $189-191{ }^{\circ} \mathrm{C}$; IR (KBr) $v_{\max } 3041,2936(\mathrm{C}-\mathrm{H}), 1600(\mathrm{C}=\mathrm{N}) \mathrm{cm}^{-1} ;{ }^{1} \mathrm{H}-\mathrm{NMR}\left(\mathrm{DMSO}-d_{6}\right) \delta 3.19(\mathrm{~m}, 1 \mathrm{H}, \mathrm{CH}), 3.56(\mathrm{~m}, 1 \mathrm{H}$, $\mathrm{CH}), 5.82(\mathrm{~m}, 1 \mathrm{H}, \mathrm{CH}), 7.21-7.85(\mathrm{~m}, 6 \mathrm{H}, \mathrm{Ar}-\mathrm{H}), 8.47$ (s, 2H, $\left.\mathrm{NH}_{2}\right), \mathrm{ppm}$; MS m/z (\%) $356\left(\mathrm{M}^{+}, 17\right)$, 280 (49), 193 (27), 104 (30), 76 (82), 41 (100). Anal. Calcd for $\mathrm{C}_{14} \mathrm{H}_{11} \mathrm{Cl}_{2} \mathrm{~N}_{3} \mathrm{~S}_{2}$ (356.29): C, 47.19; H, 3.11; N, 11.79. Found: C, 47.05; H, 3.03; N, 11.58\%.

General method for the synthesis of pyrazolylthiazoles $\mathbf{5 a}-\mathbf{f}, \mathbf{8 a}-\mathbf{e}$ and $\mathbf{1 1 a}-\mathbf{f}$. A mixture of the appropriate hydrazonoyl chlorides 3 or 6 or $\alpha$-haloketones 9 ( $1 \mathrm{mmol}$ ) and the pyrazole-1-carbothioamide $2(0.233 \mathrm{~g}, 1 \mathrm{mmol})$ was taken in a mortar at room temperature. A catalytic amount of DABCO was added. The reaction mixture was ground by the pestle under the hood for 10-20 min (monitored through TLC). The reaction mixture was then poured into water, and the solid product was collected by filtration. The crude product was recrystallized from the appropriate solvent to give the corresponding pyrazolylthiazoles $\mathbf{5 a}-\mathbf{f}, \mathbf{8 a}-\mathbf{e}$ and $\mathbf{1 1 a}-\mathbf{f}$, respectively. The products together with their physical data are listed below.

2-(5-(2,4-Dichlorophenyl)-3-(thiophen-2-yl)-4,5-dihydro-1H-pyrazol-1-yl)-4-methyl-5-(phenyldiazenyl)thiazole (5a). Red color, 72\% yield, m.p. 135-136 ${ }^{\circ} \mathrm{C}(\mathrm{EtOH}) ; \mathrm{IR}(\mathrm{KBr}) \mathrm{v}_{\max } 3054,2917(\mathrm{C}-\mathrm{H}), 1601$ (C=N) $\mathrm{cm}^{-1},{ }^{1} \mathrm{H}-\mathrm{NMR}\left(\mathrm{DMSO}-d_{6}\right) \delta 2.47\left(\mathrm{~s}, 3 \mathrm{H}, \mathrm{CH}_{3}\right), 3.14(\mathrm{~m}, 1 \mathrm{H}, \mathrm{CH}), 3.54(\mathrm{~m}, 1 \mathrm{H}, \mathrm{CH}), 5.95(\mathrm{~m}, 1 \mathrm{H}, \mathrm{CH})$, 7.05-8.05 (m, 11H, Ar-H) ppm; ${ }^{13} \mathrm{C}-\mathrm{NMR}$ NMR (DMSO-d 6$) \delta 16.0\left(\mathrm{CH}_{3}\right), 43.3\left(\mathrm{CH}_{2}\right), 56.8(\mathrm{CH}), 122.12$, $123.98,128.34,128.51,128.62,129.19,129.76,130.2,130.3,130.20,130.48,137.26,41.24,142.23,143.15$, 144.13, 152.58, 158.26, 164.42 (Ar-C and C=N) ppm; MS m/z (\%) $498\left(\mathrm{M}^{+}, 8\right), 482(40), 347(37), 319(62)$, 280 (78), 233 (53), 175 (30), 126 (39), 76 (58), 54 (45), 41 (100). Anal. Calcd for $\mathrm{C}_{23} \mathrm{H}_{17} \mathrm{Cl}_{2} \mathrm{~N}_{5} \mathrm{~S}_{2}$ (498.44): C, 55.42; H, 3.44; N, 14.05. Found: C, 55.65; H, 3.26; N, 13.91\%.

2-(5-(2,4-Dichlorophenyl)-3-(thiophen-2-yl)-4,5-dihydro-1H-pyrazol-1-yl)-4-methyl-5-(p-tolyldiazenyl)thiazole (5b). Red color, 74\% yield, m.p. 137-139 ${ }^{\circ} \mathrm{C}(\mathrm{EtOH})$; IR (KBr) v $\max 3120,2917(\mathrm{C}-\mathrm{H}), 1609(\mathrm{C}=\mathrm{N}) \mathrm{cm}^{-1}$; ${ }^{1} \mathrm{H}-\mathrm{NMR}\left(\mathrm{DMSO}-d_{6}\right) \delta 2.16\left(\mathrm{~s}, 3 \mathrm{H}, \mathrm{CH}_{3}\right), 2.29\left(\mathrm{~s}, 3 \mathrm{H}, \mathrm{CH}_{3}\right), 3.05(\mathrm{~m}, 1 \mathrm{H}, \mathrm{CH}), 3.57(\mathrm{~m}, 1 \mathrm{H}, \mathrm{CH}), 5.99$ $(\mathrm{m}, 1 \mathrm{H}, \mathrm{CH}), 7.07-8.95(\mathrm{~m}, 10 \mathrm{H}, \mathrm{Ar}-\mathrm{H}) \mathrm{ppm} ;{ }^{13} \mathrm{C}-\mathrm{NMR}\left(\mathrm{DMSO}-d_{6}\right) \delta 16.29,20.31\left(\mathrm{CH}_{3}\right), 43.0\left(\mathrm{CH}_{2}\right)$, $57.93(\mathrm{CH}), 119.30,121.24,125.81,128.17,128.74,129.11,130.38,130.90,131.05,131.62,134.29,137.02$, 140.34, 140.86, 142.58, 144.06, 157.16, 164.05 (Ar-C and C=N) ppm; MS m/z (\%) $512\left(\mathrm{M}^{+}, 20\right), 484(35)$, 
423 (20), 392 (82), 320 (76), 289 (64), 249 (48), 141 (53), 104 (48), 76 (89), 64 (100), 50 (97). Anal. Calcd for $\mathrm{C}_{24} \mathrm{H}_{19} \mathrm{Cl}_{2} \mathrm{~N}_{5} \mathrm{~S}_{2}$ (512.47): $\mathrm{C}, 56.25 ; \mathrm{H}, 3.74 ; \mathrm{N}, 13.67$. Found: $\mathrm{C}, 56.45 ; \mathrm{H}, 3.65 ; \mathrm{N}, 13.45 \%$.

2-(5-(2,4-Dichlorophenyl)-3-(thiophen-2-yl)-4,5-dihydro-1H-pyrazol-1-yl)-5-((4-methoxyphenyl) diazenyl)-4 -methylthiazole (5c). Red color, 71\% yield, m.p. 130-132 ${ }^{\circ} \mathrm{C}(\mathrm{EtOH})$; IR (KBr) $\mathrm{v}_{\max }$ 3068, 2925 (C-H), $1593(\mathrm{C}=\mathrm{N}) \mathrm{cm}^{-1}{ }^{1}{ }^{1} \mathrm{H}-\mathrm{NMR}$ (DMSO- $\left.d_{6}\right) \delta 2.27\left(\mathrm{~s}, 3 \mathrm{H}, \mathrm{CH}_{3}\right), 3.05(\mathrm{~m}, 1 \mathrm{H}, \mathrm{CH}), 3.57(\mathrm{~m}, 1 \mathrm{H}, \mathrm{CH}), 3.75$ $\left(\mathrm{s}, 3 \mathrm{H}, \mathrm{OCH}_{3}\right), 6.03(\mathrm{~m}, 1 \mathrm{H}, \mathrm{CH}), 6.94-8.59(\mathrm{~m}, 10 \mathrm{H}, \mathrm{Ar}-\mathrm{H}) \mathrm{ppm}$; MS m/z (\%) $528\left(\mathrm{M}^{+}, 9\right), 472(10)$, 365 (30), 320 (23), 267 (53), 239 (28), 161 (62), 133 (100), 117 (48), 104 (68), 78 (40), 57 (42). Anal. Calcd for $\mathrm{C}_{24} \mathrm{H}_{19} \mathrm{Cl}_{2} \mathrm{~N}_{5} \mathrm{OS}_{2}$ (528.48): C, 54.54; $\mathrm{H}, 3.62 ; \mathrm{N}, 13.25$. Found: $\mathrm{C}, 54.76 ; \mathrm{H}, 3.45 ; \mathrm{N}, 13.11 \%$.

5-((4-Chlorophenyl)diazenyl)-2-(5-(2,4-dichlorophenyl)-3-(thiophen-2-yl)-4,5-dihydro-1H-pyrazol-1-yl)-4methylthiazole (5d). Red color, 67\% yield, m.p. $120-122{ }^{\circ} \mathrm{C}(\mathrm{EtOH}) ; \mathrm{IR}(\mathrm{KBr}) \mathrm{v}_{\max } 3081,2921$ (C-H), 1600 $(\mathrm{C}=\mathrm{N}) \mathrm{cm}^{-1} ;{ }^{1} \mathrm{H}-\mathrm{NMR}\left(\mathrm{DMSO}-d_{6}\right) \delta 2.42\left(\mathrm{~s}, 3 \mathrm{H}, \mathrm{CH}_{3}\right), 3.04(\mathrm{~m}, 1 \mathrm{H}, \mathrm{CH}), 3.57(\mathrm{~m}, 1 \mathrm{H}, \mathrm{CH}), 6.06(\mathrm{~m}, 1 \mathrm{H}$, $\mathrm{CH}), 7.07-8.94$ (m, 10H, Ar-H) ppm; MS m/z (\%) 532 (M+10), 518 (34), 493 (51), 409 (99), 383 (84), 347 (33), 281 (59), 198 (60), 76 (30), 43 (100). Anal. Calcd for $\mathrm{C}_{23} \mathrm{H}_{16} \mathrm{Cl}_{3} \mathrm{~N}_{5} \mathrm{~S}_{2}$ (532.89): C, 51.84; $\mathrm{H}, 3.03$; $\mathrm{N}, 13.14$. Found: $\mathrm{C}, 52.11 ; \mathrm{H}, 2.91 ; \mathrm{N}, 12.92 \%$.

5-((4-Bromophenyl)diazenyl)-2-(5-(2,4-dichlorophenyl)-3-(thiophen-2-yl)-4,5-dihydro-1H-pyrazol-1-yl)-4methylthiazole (5e). Red color, 75\% yield, m.p. 143-145 ${ }^{\circ} \mathrm{C}$ (Dioxane); IR (KBr) v $v_{\max } 3147,2969$ (C-H), $1604(\mathrm{C}=\mathrm{N}) \mathrm{cm}^{-1},{ }^{1} \mathrm{H}-\mathrm{NMR}\left(\mathrm{DMSO}-d_{6}\right) \delta 2.49\left(\mathrm{~s}, 3 \mathrm{H}, \mathrm{CH}_{3}\right), 3.07(\mathrm{~m}, 1 \mathrm{H}, \mathrm{CH}), 3.52(\mathrm{~m}, 1 \mathrm{H}, \mathrm{CH}), 6.01$ (m, 1H, CH), 7.40-8.42 (m, 10H, Ar-H) ppm; MS m/z (\%) $577\left(\mathrm{M}^{+}, 7\right), 523$ (24), 439 (21), 332 (58), 299 (34), 146 (57), 110 (28), 68 (100), 42 (77). Anal. Calcd for $\mathrm{C}_{23} \mathrm{H}_{16} \mathrm{BrCl}_{2} \mathrm{~N}_{5} \mathrm{~S}_{2}$ (577.34): C, 47.85; H, $2.79 ; \mathrm{N}, 12.13$. Found: $\mathrm{C}, 48.07 ; \mathrm{H}, 2.65 ; \mathrm{N}, 12.01 \%$.

2-(5-(2,4-Dichlorophenyl)-3-(thiophen-2-yl)-4,5-dihydro-1H-pyrazol-1-yl)-4-methyl-5-((4-nitrophenyl)diazenyl) thiazole (5f). Orange color, 68\% yield, m.p. 140-141 ${ }^{\circ} \mathrm{C}$ (Dioxane); IR (KBr) $\mathrm{v}_{\max } 3084,2938(\mathrm{C}-\mathrm{H})$, $1605(\mathrm{C}=\mathrm{N}) \mathrm{cm}^{-1} ;{ }^{1} \mathrm{H}-\mathrm{NMR}\left(\mathrm{DMSO}-d_{6}\right) \delta 2.43\left(\mathrm{~s}, 3 \mathrm{H}, \mathrm{CH}_{3}\right), 3.04(\mathrm{~m}, 1 \mathrm{H}, \mathrm{CH}), 3.54(\mathrm{~m}, 1 \mathrm{H}, \mathrm{CH}), 6.05$ (m, 1H, CH), 7.30-7.99 (m, 10H, Ar-H) ppm; MS m/z (\%) $543\left(\mathrm{M}^{+}, 12\right), 485$ (27), 453 (31), 394 (100), 318 (39), 258 (26), 244 (33), 160 (37), 107 (54), 81 (65), 43 (91). Anal. Calcd for $\mathrm{C}_{23} \mathrm{H}_{16} \mathrm{Cl}_{2} \mathrm{~N}_{6} \mathrm{O}_{2} \mathrm{~S}_{2}$ (543.44): C, 50.83; H, 2.97; N, 15.46. Found: C, 51.12, H, 2.75; N, 15.27\%.

2-(5-(2,4-Dichlorophenyl)-3-(thiophen-2-yl)-4,5-dihydro-1H-pyrazol-1-yl)-5-(2-phenylhydrazono) thiazol-4 (5H)-one (8a). Yellow color, 70\% yield, m.p.145-147 ${ }^{\circ} \mathrm{C}(\mathrm{EtOH}) ; \mathrm{IR}(\mathrm{KBr}) \mathrm{v}_{\max } 3433(\mathrm{NH}), 3093$, $2923(\mathrm{C}-\mathrm{H}), 1686(\mathrm{C}=\mathrm{O}), 1612(\mathrm{C}=\mathrm{N}) \mathrm{cm}^{-1},{ }^{1} \mathrm{H}-\mathrm{NMR}\left(\mathrm{DMSO}-d_{6}\right) \delta 3.03(\mathrm{~m}, 1 \mathrm{H}, \mathrm{CH}), 3.56(\mathrm{~m}, 1 \mathrm{H}$, $\mathrm{CH}), 5.96(\mathrm{~m}, 1 \mathrm{H}, \mathrm{CH}), 6.94-8.69(\mathrm{~m}, 11 \mathrm{H}, \mathrm{Ar}-\mathrm{H}), 11.74(\mathrm{~s}, 1 \mathrm{H}, \mathrm{NH}) \mathrm{ppm} ; \mathrm{MS} \mathrm{m} / z(\%) 500\left(\mathrm{M}^{+}, 14\right)$, 422 (23), 396 (56), 316 (100), 276 (10), 161 (39), 104 (36), 82 (64), 67 (60), 43 (93). Anal. Calcd for $\mathrm{C}_{22} \mathrm{H}_{15} \mathrm{Cl}_{2} \mathrm{~N}_{5} \mathrm{OS}_{2}$ (500.42): C, 52.80; H, 3.02; N, 14.00. Found: $\mathrm{C}, 53.05 ; \mathrm{H}, 2.87 ; \mathrm{N}, 13.77 \%$.

2-(5-(2,4-Dichlorophenyl)-3-(thiophen-2-yl)-4,5-dihydro-1H-pyrazol-1-yl)-5-(2-(p-tolyl)hydrazono)thiazol-4 (5H)-one (8b). Yellow color, $72 \%$ yield, m.p. $163-165{ }^{\circ} \mathrm{C}$ (Dioxane); IR (KBr) $\mathrm{v}_{\max } 3434(\mathrm{NH}), 3066$, $2922(\mathrm{C}-\mathrm{H}), 1649(\mathrm{C}=\mathrm{O}), 1612(\mathrm{C}=\mathrm{N}) \mathrm{cm}^{-1} ;{ }^{13} \mathrm{C}-\mathrm{NMR}\left(\mathrm{DMSO}-d_{6}\right) \delta 20.84\left(\mathrm{CH}_{3}\right), 43.32\left(\mathrm{CH}_{2}\right), 60.47(\mathrm{CH})$, $112.53,115.07,118.93,126.11,127.76,127.95,129.67,130.13,130.62,131.20,132.27,133.32,140.70,150.36$, 151.39, 163.37, $168.10($ Ar- $\mathrm{C}$ and $\mathrm{C}=\mathrm{N}), 170.85(\mathrm{C}=\mathrm{O}) \mathrm{ppm} ;{ }^{1} \mathrm{H}-\mathrm{NMR}\left(\mathrm{DMSO}-d_{6}\right) \delta 2.27\left(\mathrm{~s}, 3 \mathrm{H}, \mathrm{CH}_{3}\right)$, $3.02(\mathrm{~m}, 1 \mathrm{H}, \mathrm{CH}), 3.58(\mathrm{~m}, 1 \mathrm{H}, \mathrm{CH}), 5.98(\mathrm{~m}, 1 \mathrm{H}, \mathrm{CH}), 7.11-8.31(\mathrm{~m}, 10 \mathrm{H}, \mathrm{Ar}-\mathrm{H}), 11.66(\mathrm{~s}, \mathrm{H}, \mathrm{NH}) \mathrm{ppm}$; MS m/z (\%) $514\left(\mathrm{M}^{+}, 20\right), 502$ (50), 469 (39), 439 (44), 320 (84), 294 (34), 158 (38), 119 (40), 77 (100), 42 (31). Anal. Calcd for $\mathrm{C}_{23} \mathrm{H}_{17} \mathrm{Cl}_{2} \mathrm{~N}_{5} \mathrm{OS}_{2}$ (514.44): C, 53.70; $\mathrm{H}, 3.33 ; \mathrm{N}, 13.61$. Found: $\mathrm{C}, 53.55 ; \mathrm{H}, 3.50$; $\mathrm{N}, 13.29 \%$.

5-(2-(4-Chlorophenyl)hydrazono)-2-(5-(2,4-dichlorophenyl)-3-(thiophen-2-yl)-4,5-dihydro-1H-pyrazol-1-yl) thiazol-4(5H)-one (8c). Yellow color, 70\% yield, m.p. 178-180 ${ }^{\circ} \mathrm{C}(\mathrm{DMF})$; IR (KBr) $\mathrm{v}_{\max } 3433(\mathrm{NH}), 3089$, $2926(\mathrm{C}-\mathrm{H}), 1649(\mathrm{C}=\mathrm{O}), 1617(\mathrm{C}=\mathrm{N}) \mathrm{cm}^{-1}{ }^{1}{ }^{1} \mathrm{H}-\mathrm{NMR}$ (DMSO-d $\left.d_{6}\right) \delta 3.05(\mathrm{~m}, 1 \mathrm{H}, \mathrm{CH}), 3.52(\mathrm{~m}, 1 \mathrm{H}, \mathrm{CH})$, $5.97(\mathrm{~m}, 1 \mathrm{H}, \mathrm{CH}), 7.08-8.62(\mathrm{~m}, 10 \mathrm{H}, \mathrm{Ar}-\mathrm{H}), 11.69$ (s, 1H, NH) ppm; MS m/z (\%) $534\left(\mathrm{M}^{+}, 12\right), 514(44)$, 456 (21), 432 (16), 327 (38), 316 (63), 297 (100), 239 (26), 135 (31), 95 (72), 68 (43), 43 (59). Anal. Calcd for $\mathrm{C}_{22} \mathrm{H}_{14} \mathrm{Cl}_{3} \mathrm{~N}_{5} \mathrm{OS}_{2}$ (534.86): $\mathrm{C}, 49.40 ; \mathrm{H}, 2.64 ; \mathrm{N}, 13.09$. Found: $\mathrm{C}, 49.62 ; \mathrm{H}, 2.43 ; \mathrm{N}, 13.25 \%$. 
2-(5-(2,4-Dichlorophenyl)-3-(thiophen-2-yl)-4,5-dihydro-1H-pyrazol-1-yl)-5-(2-(4-nitrophenyl) hydrazono) thiazol-4(5H)-one (8d). Yellow color, 74\% yield, m.p. $155-156{ }^{\circ} \mathrm{C}(\mathrm{EtOH}) ; \mathrm{IR}(\mathrm{KBr}) \mathrm{v}_{\max } 3432(\mathrm{NH}), 3079$, $2924(\mathrm{C}-\mathrm{H}), 1653(\mathrm{C}=\mathrm{O}), 1592(\mathrm{C}=\mathrm{N}) \mathrm{cm}^{-1} ;{ }^{1} \mathrm{H}-\mathrm{NMR}$ (DMSO-d $\left.)_{6}\right) \delta 3.07(\mathrm{~m}, 1 \mathrm{H}, \mathrm{CH}), 3.54(\mathrm{~m}, 1 \mathrm{H}, \mathrm{CH})$, $6.04(\mathrm{~m}, 1 \mathrm{H}, \mathrm{CH}), 7.11-8.69(\mathrm{~m}, 10 \mathrm{H}, \mathrm{Ar}-\mathrm{H}), 11.62(\mathrm{~s}, 1 \mathrm{H}, \mathrm{NH}) \mathrm{ppm}$; MS m/z (\%) $545\left(\mathrm{M}^{+}, 5\right), 502(18)$, 422 (47), 396 (39), 353 (60), 317 (100), 257 (25), 199 (21), 164 (40), 101 (52), 95 (36), 78 (42). Anal. Calcd for $\mathrm{C}_{22} \mathrm{H}_{14} \mathrm{Cl}_{2} \mathrm{~N}_{6} \mathrm{O}_{3} \mathrm{~S}_{2}$ (545.41): $\mathrm{C}, 48.45 ; \mathrm{H}, 2.59 ; \mathrm{N}, 15.41$. Found: $\mathrm{C}, 48.52 ; \mathrm{H}, 2.75 ; \mathrm{N}, 15.33 \%$.

Ethyl-4-(2-(2-(5-(2,4-dichlorophenyl)-3-(thiophen-2-yl)-4,5-dihydro-1H-pyrazol-1-yl)-4-oxothiazol-5(4H)ylidene)hydrazinyl)benzoate (8e). Yellow color, 67\% yield, m.p. 168-170 ${ }^{\circ} \mathrm{C}(\mathrm{DMF}) ; \mathrm{IR}(\mathrm{KBr}) \mathrm{v}_{\max } 3430$ $(\mathrm{NH}), 3079,2916(\mathrm{C}-\mathrm{H}), 1649(\mathrm{C}=\mathrm{O}), 1590(\mathrm{C}=\mathrm{N}) \mathrm{cm}^{-1},{ }^{1} \mathrm{H}-\mathrm{NMR}$ (DMSO-d $) \delta 1.17\left(\mathrm{t}, 3 \mathrm{H}, \mathrm{CH}_{3}\right), 3.00$ $(\mathrm{m}, 1 \mathrm{H}, \mathrm{CH}), 3.54(\mathrm{~m}, 1 \mathrm{H}, \mathrm{CH}), 4.25\left(\mathrm{q}, 2 \mathrm{H}, \mathrm{CH}_{2}\right), 5.95(\mathrm{~m}, 1 \mathrm{H}, \mathrm{CH}), 7.04-8.19(\mathrm{~m}, 10 \mathrm{H}, \mathrm{Ar}-\mathrm{H}), 11.17$ (s, 1H, NH) ppm: MS m/z (\%) $572\left(\mathrm{M}^{+}, 32\right), 509$ (31), 458 (49), 439 (100), 402 (50), 387 (56), 359 (58), 347 (62), 94 (43), 68 (52), 42 (78). Anal. Calcd for $\mathrm{C}_{25} \mathrm{H}_{19} \mathrm{Cl}_{2} \mathrm{~N}_{5} \mathrm{O}_{3} \mathrm{~S}_{2}$ (572.48): $\mathrm{C}, 52.45 ; \mathrm{H}, 3.35 ; \mathrm{N}, 12.23$. Found: C, 52.66; H, 3.17; N, 12.06\%.

2-(5-(2,4-Dichlorophenyl)-3-(thiophen-2-yl)-4,5-dihydro-1H-pyrazol-1-yl)-4-phenylthiazole (11a). Yellow color, 79\% yield, m.p. $193-195{ }^{\circ} \mathrm{C}$ (Dioxane); IR (KBr) $v_{\max } 3054,2920(\mathrm{C}-\mathrm{H}), 1609(\mathrm{C}=\mathrm{N}) \mathrm{cm}^{-1}$; ${ }^{1} \mathrm{H}-\mathrm{NMR}\left(\mathrm{DMSO}-d_{6}\right) \delta 3.01(\mathrm{~m}, 1 \mathrm{H}, \mathrm{CH}), 3.53(\mathrm{~m}, 1 \mathrm{H}, \mathrm{CH}), 5.97(\mathrm{~m}, 1 \mathrm{H}, \mathrm{CH}), 6.98-7.86(\mathrm{~m}, 11 \mathrm{H}, \mathrm{Ar}-\mathrm{H})$, 8.39 (s, 1H, thiazole-H) ppm; MS m/z (\%) $456\left(\mathrm{M}^{+}, 17\right), 427$ (56), 366 (52), 285 (35), 200 (39), 172 (100), 131 (93), 101 (38), 77 (85). Anal. Calcd for $\mathrm{C}_{22} \mathrm{H}_{15} \mathrm{Cl}_{2} \mathrm{~N}_{3} \mathrm{~S}_{2}$ (456.41): C, 57.89; $\mathrm{H}, 3.31 ; \mathrm{N}, 9.21$. Found: C, $57.72 ; \mathrm{H}, 3.25 ; \mathrm{N}, 9.10 \%$.

2-(5-(2,4-Dichlorophenyl)-3-(thiophen-2-yl)-4,5-dihydro-1H-pyrazol-1-yl)-4-(p-tolyl)thiazole (11b). Yellow brown, 78\% yield, m.p. $184-186{ }^{\circ} \mathrm{C}$ (Dioxane); IR (KBr) $v_{\max } 3032,2915(\mathrm{C}-\mathrm{H}), 1608(\mathrm{C}=\mathrm{N}) \mathrm{cm}^{-1}$; ${ }^{1} \mathrm{H}-\mathrm{NMR}\left(\mathrm{DMSO}-d_{6}\right) \delta 2.27\left(\mathrm{~s}, 3 \mathrm{H}, \mathrm{CH}_{3}\right), 3.01(\mathrm{~m}, 1 \mathrm{H}, \mathrm{CH}), 3.47(\mathrm{~m}, 1 \mathrm{H}, \mathrm{CH}), 6.03(\mathrm{~m}, 1 \mathrm{H}, \mathrm{CH})$, 6.95-8.00 (m, 10H, Ar-H), 8.35 (s, $1 \mathrm{H}$, thiazole-H) ppm; ${ }^{13} \mathrm{C}-\mathrm{NMR}$ (DMSO- $\left.d_{6}\right) \delta 19.01\left(\mathrm{CH}_{3}\right), 43.74\left(\mathrm{CH}_{2}\right)$, $60.22(\mathrm{CH}), 103.81,125.98,128.21,128.42,129.34,129.52,129.65,132.11,133.25,133.98,134.53,135.53$, $136.48,137.33,137.44,144.11,166.89,168.12(\mathrm{Ar}-\mathrm{C}$ and $\mathrm{C}=\mathrm{N}) \mathrm{ppm}$; MS $m / z(\%) 468\left(\mathrm{M}^{+}-2,10\right), 418(24)$, 399 (59), 353 (74), 285 (59), 204 (45), 184 (49), 92 (70), 44 (100). Anal. Calcd for $\mathrm{C}_{23} \mathrm{H}_{17} \mathrm{Cl}_{2} \mathrm{~N}_{3} \mathrm{~S}_{2}$ (470.44): C, $58.72 ; \mathrm{H}, 3.64 ; \mathrm{N}, 8.93$. Found: $\mathrm{C}, 58.79 ; \mathrm{H}, 3.52 ; \mathrm{N}, 8.75 \%$.

2-(5-(2,4-Dichlorophenyl)-3-(thiophen-2-yl)-4,5-dihydro-1H-pyrazol-1-yl)-4-(4-methoxyphenyl)thiazole (11c). Yellow color, 71\% yield, m.p.168-170 ${ }^{\circ} \mathrm{C}(\mathrm{EtOH})$; IR $(\mathrm{KBr}) \mathrm{v}_{\max } 3088,2931(\mathrm{C}-\mathrm{H}), 1591(\mathrm{C}=\mathrm{N}) \mathrm{cm}^{-1}$; ${ }^{1} \mathrm{H}-\mathrm{NMR}\left(\mathrm{DMSO}-d_{6}\right) \delta 3.04(\mathrm{~m}, 1 \mathrm{H}, \mathrm{CH}), 3.57(\mathrm{~m}, 1 \mathrm{H}, \mathrm{CH}), 3.79\left(\mathrm{~s}, 3 \mathrm{H}, \mathrm{OCH}_{3}\right), 5.99(\mathrm{~m}, 1 \mathrm{H}, \mathrm{CH})$, 6.89-8.37 (m, 10H, Ar-H), $8.42\left(\mathrm{~s}, 1 \mathrm{H}\right.$, thiazole-H) ppm. MS m/z (\%) $486\left(\mathrm{M}^{+}, 13\right), 420$ (19), $245(23)$, 170 (54), 92 (100), 83 (16), 72 (5). Anal. Calcd for $\mathrm{C}_{23} \mathrm{H}_{17} \mathrm{Cl}_{2} \mathrm{~N}_{3} \mathrm{OS}_{2}$ (486.44): C, 56.79; $\mathrm{H}, 3.52 ; \mathrm{N}, 8.64$. Found: $\mathrm{C}, 56.73 ; \mathrm{H}, 3.45 ; \mathrm{N}, 8.33 \%$.

4-(4-Chlorophenyl)-2-(5-(2,4-dichlorophenyl)-3-(thiophen-2-yl)-4,5-dihydro-1H-pyrazol-1-yl)thiazole (11d). Yellow color, 75\% yield, m.p. 189-191 ${ }^{\circ} \mathrm{C}$ (Dioxane); IR (KBr) $\mathrm{v}_{\max }$ 3086, $2925(\mathrm{C}-\mathrm{H}), 1611(\mathrm{C}=\mathrm{N}) \mathrm{cm}^{-1}$; ${ }^{1} \mathrm{H}-\mathrm{NMR}$ (DMSO-d $\left.d_{6}\right) \delta 3.02(\mathrm{~m}, 1 \mathrm{H}, \mathrm{CH}), 3.54(\mathrm{~m}, 1 \mathrm{H}, \mathrm{CH}), 5.97(\mathrm{~m}, 1 \mathrm{H}, \mathrm{CH}), 6.951-8.00(\mathrm{~m}, 10 \mathrm{H}, \mathrm{Ar}-\mathrm{H})$, $8.36\left(\mathrm{~s}, 1 \mathrm{H}\right.$, thiazole-H) ppm. MS $m / z(\%) 490\left(\mathrm{M}^{+}, 8\right), 455$ (38), 424 (92), 404 (54), 320 (100), 186 (79), 132 (46), 75 (51). Anal. Calcd for $\mathrm{C}_{22} \mathrm{H}_{14} \mathrm{Cl}_{3} \mathrm{~N}_{3} \mathrm{~S}_{2}$ (490.86): C, 53.83; $\mathrm{H}, 2.87 ; \mathrm{N}, 8.56$. Found: $\mathrm{C}, 53.69$; $\mathrm{H}, 3.04 ; \mathrm{N}, 8.39 \%$.

4-(4-Bromophenyl)-2-(5-(2,4-dichlorophenyl)-3-(thiophen-2-yl)-4,5-dihydro-1H-pyrazol-1-yl)thiazole (11e). Yellow color, 74\% yield, m.p. 182-184 ${ }^{\circ} \mathrm{C}$ (Dioxane); IR (KBr) $\mathrm{v}_{\max }$ 3068, $2920(\mathrm{C}-\mathrm{H}), 1611(\mathrm{C}=\mathrm{N})$ $\mathrm{cm}^{-1} ;{ }^{1} \mathrm{H}-\mathrm{NMR}$ (DMSO- $\left.d_{6}\right) \delta 3.04(\mathrm{~m}, 1 \mathrm{H}, \mathrm{CH}), 3.48(\mathrm{~m}, 1 \mathrm{H}, \mathrm{CH}), 5.95(\mathrm{~m}, 1 \mathrm{H}, \mathrm{CH}), 6.97-8.30(\mathrm{~m}, 10 \mathrm{H}$, Ar-H), 8.32 (s, 1H, thiazole-H5) ppm; MS m/z (\%) 535 (M+1 11), 524 (43), 511 (94), 472 (92), 432 (100), 404 (93), 352 (47), 312 (48), 184 (41), 76 (75), 57 (51). Anal. Calcd for $\mathrm{C}_{22} \mathrm{H}_{14} \mathrm{BrCl}_{2} \mathrm{~N}_{3} \mathrm{~S}_{2}$ (535.31): C, 49.36; $\mathrm{H}, 2.64 ; \mathrm{N}, 7.85$. Found: $\mathrm{C}, 49.29 ; \mathrm{H}, 2.50 ; \mathrm{N}, 7.65 \%$.

2-(5-(2,4-Dichlorophenyl)-3-(thiophen-2-yl)-4,5-dihydro-1H-pyrazol-1-yl)-4-(4-fluorophenyl)thiazole (11f). $75 \%$ yield, m.p. $175-177{ }^{\circ} \mathrm{C}(\mathrm{EtOH}) ; \mathrm{IR}(\mathrm{KBr}) \mathrm{v}_{\max }$ 3073, $2925(\mathrm{C}-\mathrm{H}), 1591(\mathrm{C}=\mathrm{N}) \mathrm{cm}^{-1} ;{ }^{1} \mathrm{H}-\mathrm{NMR}$ 
$\left(\mathrm{DMSO}_{6}\right) \delta 3.11(\mathrm{~m}, 1 \mathrm{H}, \mathrm{CH}), 3.51(\mathrm{~m}, 1 \mathrm{H}, \mathrm{CH}), 5.91(\mathrm{~m}, 1 \mathrm{H}, \mathrm{CH}), 7.18-8.35(\mathrm{~m}, 10 \mathrm{H}, \mathrm{Ar}-\mathrm{H}), 8.42(\mathrm{~s}, 1 \mathrm{H}$, thiazole-H) ppm; MS m/z (\%) $474\left(\mathrm{M}^{+}, 14\right), 436$ (33), 408 (45), 399 (60), 368 (100), 313 (89), 264 (56), 143 (31), 68 (59). Anal. Calcd for $\mathrm{C}_{22} \mathrm{H}_{14} \mathrm{Cl}_{2} \mathrm{FN}_{3} \mathrm{~S}_{2}$ (474.40): C, 55.70; H, 2.97; N, 8.86. Found: C, 55.65; $\mathrm{H}, 2.88 ; \mathrm{N}, 8.64 \%$.

2-(5-(2,4-Dichlorophenyl)-3-(thiophen-2-yl)-4,5-dihydro-1H-pyrazol-1-yl)-4-(4-nitrophenyl)thiazole (11g). Brown color, 82\% yield, m.p. 205-207 ${ }^{\circ} \mathrm{C}(\mathrm{DMF})$; IR (KBr) vmax 3145, $2934(\mathrm{C}-\mathrm{H}), 1590(\mathrm{C}=\mathrm{N}) \mathrm{cm}^{-1}$; ${ }^{1} \mathrm{H}-\mathrm{NMR}\left(\mathrm{DMSO}-d_{6}\right) \delta 3.14(\mathrm{~m}, 1 \mathrm{H}, \mathrm{CH}), 3.64(\mathrm{~m}, 1 \mathrm{H}, \mathrm{CH}), 5.86(\mathrm{~m}, 1 \mathrm{H}, \mathrm{CH}), 7.24-8.38(\mathrm{~m}, 10 \mathrm{H}, \mathrm{Ar}-\mathrm{H})$, 8.42 (s, 1H, thiazole-H) ppm; MS m/z (\%) $501\left(\mathrm{M}^{+}, 18\right), 487$ (46), 461 (74), 425 (100), 396 (98), 315 (49), 287 (43), 223 (13), 166 (25), 130 (23). Anal. Calcd for $\mathrm{C}_{22} \mathrm{H}_{14} \mathrm{Cl}_{2} \mathrm{~N}_{4} \mathrm{O}_{2} \mathrm{~S}_{2}$ (501.41): C, 52.70; H, 2.81; N, 11.17. Found: C, 52.64; H, 2.73; N, 11.00\%.

4-(2-(5-(2,4-Dichlorophenyl)-3-(thiophen-2-yl)-4,5-dihydro-1H-pyrazol-1-yl)thiazol-4-yl)aniline (11h). Brown color, 78\% yield, m.p. $184-186{ }^{\circ} \mathrm{C}$ (Dioxane); IR (KBr) v $\max 3394-3264\left(\mathrm{NH}_{2}\right), 3147,2935$ (C-H), $1690(\mathrm{C}=\mathrm{N}) \mathrm{cm}^{-1},{ }^{1} \mathrm{H}-\mathrm{NMR}\left(\mathrm{DMSO}-d_{6}\right) \delta 3.01(\mathrm{~m}, 1 \mathrm{H}, \mathrm{CH}), 3.55(\mathrm{~m}, 1 \mathrm{H}, \mathrm{CH}), 6.00(\mathrm{~m}, 1 \mathrm{H}, \mathrm{CH})$, 6.08 (br s, 2H, $\left.\mathrm{NH}_{2}\right), 6.95-8.00(\mathrm{~m}, 10 \mathrm{H}, \mathrm{Ar}-\mathrm{H}), 8.65$ (s, 1H, thiazole-H) ppm; MS m/z (\%) $471\left(\mathrm{M}^{+}, 28\right)$, 461 (100), 433 (66), 397 (64), 366 (75), 306 (17), 298 (17), 141 (26), 70 (93). Anal. Calcd for $\mathrm{C}_{22} \mathrm{H}_{16} \mathrm{Cl}_{2} \mathrm{~N}_{4} \mathrm{~S}_{2}$ (471.43): C, 56.05; H, 3.42; N, 11.88. Found: C, 55.99; H, 3.67; N, 11.67\%.

\subsection{Cytotoxic Activity.}

The cytotoxic activity of the synthesized compounds was evaluated against the liver Carcinoma (HepG2) cell line at the Regional Center for Mycology and Biotechnology at Al-Azhar University, Cairo, Egypt according to the reported methods [37,38]. For more details, see the supporting information file.

\subsection{Antimicrobial Evaluation.}

Agar Diffusion Well Method [39,40] was used to determine the antimicrobial activity of the synthesized compounds. For more details, see the supporting information file.

\section{Conclusions}

We used an eco-friendly method for the synthesis of a novel series of pyrazolines containing the bioactive thiazole moiety. The structures of the newly synthesized compounds were established based on both elemental and spectroscopic analysis. The cytotoxic activity against liver Carcinoma (HepG2) cell line was measured and it showed that the pyrazoline derivatives 11f, 11d and 8c had $\mathrm{IC}_{50}$ values of $1.7,2.98$ and $3.54 \mu \mathrm{M}$, respectively. The results of the antifungal evaluation revealed that the pyrazolines 11a, 11b, 11d, and 11e are the most potent compounds against Aspergillus fumigatus and the pyrazoline 11a is more potent than the standard drug Ketoconazole against Candida albicans. The results of antibacterial evaluation showed that the pyrazoline 11a is more potent than the standard drug Gentamycin against Staphylococcus aureus and Proteus vulgaris.

Supplementary Materials: The following are available online, Methods of the cytotoxic, and antimicrobial evaluation, Figures (Figures S1-S5) of mean zone of inhibition, and the NMRs of the new synthesized compounds.

Author Contributions: M.M.E., S.A.M., N.A.K. and S.M.G. conceived and designed the experiments; M.M.E. and Z.A.M. carried out the experiments; S.A.M., M.M.E. and A.M.S. analyzed and interpreted the data; S.M.G., N.A.K. and S.A.M. prepared the manuscript. All authors have read and approved the final manuscript.

Funding: The authors extend their appreciation to the Deanship of Scientific Research at King Khalid University for funding this work through a General Research Project under grant number (GRP-68-39).

Conflicts of Interest: The authors declare no conflict of interest. 


\section{References}

1. Mansoori, B.; Mohammadi, A.; Davudian, S.; Shirjang, S.; Baradaran, B. The Different mechanisms of cancer drug resistance: A brief review. Adv. Pharm. Bull. 2017, 7, 339-348. [CrossRef] [PubMed]

2. Gomha, S.M.; Abdelhamid, A.O.; Abdelrehem, N.A.; Kandeel, S.M. Efficient Synthesis of new benzofuran-based thiazoles and investigation of their cytotoxic activity against human breast carcinoma cell lines. J. Heterocycl. Chem. 2018, 55, 995-1001. [CrossRef]

3. Gomha, S.M.; Abdallah, M.A.; Al-Showiman, S.S.; Morad, M.A.; Mabkhot, Y.N. Synthesis of new pyridopyrimidinone-based thiadiazoles and pyrazolines as potential anti-breast cancer agents. Biomedical Res. 2017, 28, 9903-9909.

4. Gomha, S.M.; Salah, T.A.; Abdelhamid, A.O. Synthesis, characterization and pharmacological evaluation of some novel thiadiazoles and thiazoles incorporating pyrazole moiety as potent anticancer agents. Monatsh. Chem. 2015, 146, 149-158. [CrossRef]

5. Gomha, S.M.; Edrees, M.M.; Altalbawy, F.M.A. Synthesis and characterization of some new bis-pyrazolyl-thiazoles incorporating the thiophene moiety as potent anti-tumor agents. Inter. J. Mol. Sci. 2016, 17, 1499. [CrossRef] [PubMed]

6. Babu, V.H.; Sridevi, C.H.; Joseph, A.; Srinivasan, K.K. Synthesis and biological evaluation of some novel pyrazolines. Indian J. Pharm. Sci. 2007, 69, 470-473.

7. Gomha, S.M.; Farghaly, T.A.; Sayed, A.R. Synthesis of pyrazolyl pyrazoles and pyrazolyl-[1,2,4]-triazolo [1,5]benzothiazepines as antimicrobial agents using hydrazonoyl chlorides. J. Heterocycl. Chem. 2016, 53, 1503-1509. [CrossRef]

8. Gomha, S.M.; Farghaly, T.A.; Mabkhot, Y.N.; Zayed, M.E.M.; Mohamed, A.M.G. Microwave-assisted synthesis of some novel azoles and azolopyrimidines as antimicrobial agents. Molecules 2017, $22,346$. [CrossRef] [PubMed]

9. Taj, T.; Kamble, R.R.; Gireesh, T.M.; Hunnur, R.K.; Margankop, S.B. One-pot synthesis of pyrazoline derivatised carbazoles as antitubercular, anticancer agents, their DNA cleavage and antioxidant activities. Eur. J. Med. Chem. 2011, 46, 4366-4373. [CrossRef] [PubMed]

10. Wanare, G.; Aher, R.; Kawathekar, N.; Ranjan, R.; Kaushik, N.K.; Sahal, D. Synthesis of novel $\alpha$-pyranochalcones and pyrazoline derivatives as Plasmodium falciparum growth inhibitors. Bioorg. Med. Chem. Lett. 2010, 20, 4675-4678. [CrossRef] [PubMed]

11. Bhat, A.R.; Athar, F.; Azam, A. Bis-pyrazolines: Synthesis, characterization and antiamoebic activity as inhibitors of growth of Entamoeba histolytica. Eur. J. Med. Chem. 2009, 44, 426-431. [CrossRef] [PubMed]

12. Gomha, S.M.; Riyadh, S.M.; Abdalla, M.M. Solvent-drop grinding method: Efficient synthesis, DPPH radical scavenging and anti-diabetic activities of chalcones, bis-chalcones, azolines, and bis-azolines. Curr. Org. Synth. 2015, 12, 220-228. [CrossRef]

13. Gomha, S.M.; Abdalla, M.A.; Abdelaziz, M.; Serag, N. Eco-friendly one-pot synthesis and antiviral evaluation of pyrazolyl chalcones and pyrazolines of medicinal interest. Turk. J. Chem. 2016, 40, 484-498. [CrossRef]

14. Manna, F.; Chimenti, F.; Bolasco, A.; Secci, D.; Bizzarri, B.; Befani, O.; Turini, P.; Mondovi, B.; Alcaro, S.; Tafi, A. Inhibition of amine oxidases activity by 1-acetyl-3,5-diphenyl-4,5-dihydro-(1H)-pyrazole derivatives. Bioorg Med Chem Lett. 2002, 12, 3629-3633. [CrossRef]

15. Satoh, A.; Nagatomi, Y.; Hirata, Y.; Ito, S.; Suzuki, G.; Kimura, T.; Maehara, S.; Hikichi, H.; Satow, A.; Hata, M.; et al. Discovery and in vitro and invivo profiles of 4-fluoro- $N$-[4-[6-(isopropylamino)pyrimidin-4-yl]-1,3-thiazol-2-yl]- $N$-methylbenzamide as novel class of an orally active metabotropic glutamate receptor 1 (mGluR1) antagonist. Bioorg Med Chem Lett. 2009, 19, 5464-5468. [CrossRef] [PubMed]

16. Dawane, B.S.; Konda, S.G.; Mandawad, G.G.; Shaikh, B.M. Poly(ethylene glycol) (PEG-400) as an alternative reaction solvent for the synthesis of some new 1-(4-(4'-chlorophenyl)-2-thiazolyl)3-aryl-5-(2-butyl-4-chloro-1H-imidazol-5yl)-2-pyrazolines and their in vitro antimicrobial evaluation. Eur. J. Med. Chem. 2010, 45, 387-392. [CrossRef] [PubMed]

17. Sondhi, S.M.; Singh, N.; Lahoti, A.M.; Bajaj, K.; Kumar, A.; Lozach, O.; Meijer, L. Synthesis of acridinyl-thiazolino derivatives and their evaluation for anti-inflammatory, analgesic and kinase inhibition activities. Bioorg. Med. Chem. 2005, 13, 4291-4299. [CrossRef] [PubMed] 
18. Luzina, E.L.; Popov, A.V. Synthesis and anticancer activity of $N$-bis(trifluoromethyl)alkyl- $N^{\prime}$-thiazolyl and $N$-bis(trifluoromethyl)alkyl- $N^{\prime}$-benzothiazolyl ureas. Eur. J. Med. Chem. 2009, 44, 4944-4953. [CrossRef] [PubMed]

19. Gomha, S.M.; Riyadh, S.M.; Abbas, I.M.; Bauomi, M.A. Synthetic utility of ethylidenethiosemicarbazide: Synthesis and anti-cancer activity of 1,3-thiazines and thiazoles with imidazole moiety. Heterocycles 2013, 87, 341-356.

20. Gomha, S.M.; Kheder, N.A.; Abdelaziz, M.R.; Mabkhot, Y.N.; Alhajoj, A.M. A facile synthesis and anticancer activity of some novel thiazoles carrying 1,3,4-thiadiazole moiety. Chem. Cent. J. 2017, 11, 25. [CrossRef] [PubMed]

21. Iino, T.; Hashimoto, N.; Sasaki, K.; Ohyama, S.; Yoshimoto, R.; Hosaka, H.; Hasegawa, T.; Chiba, M.; Nagata, Y.; Nishimura, J.E.T. Structure activity relationships of 3,5-disubstituted benzamides as glucokinase activators with potent in vivo efficacy. Bioorg. Med. Chem. 2009, 17, 3800-3809. [CrossRef] [PubMed]

22. Rawal, R.K.; Tripathi, R.; Katti, S.B.; Pannecouque, C.; Clercq, E.D. Design and synthesis of 2-(2,6-dibromophenyl)-3-heteroaryl-1,3-thiazolidin-4-ones as anti-HIV agents. Eur J Med. Chem. 2008, 43, 2800-2806. [CrossRef] [PubMed]

23. Shiradkar, M.R.; Akula, K.C.; Dasari, V.; Baru, V.; Chiningiri, B.; Gandhi, S.; Kaur, R. Clubbed thiazoles by MAOS: a novel approach to cyclin-dependent kinase 5/p25 inhibitors as a potential treatment for Alzheimer's disease. Bioorg. Med. Chem. 2007, 15, 2601-2610. [CrossRef] [PubMed]

24. Turan-Zitouni, G.; Chevallet, P.; Kiliç, F.S.; Erol, K. Synthesis of some thiazolyl-pyrazoline derivatives and preliminary investigation of their hypotensive activity. Eur. J. Med. Chem. 2000, 35, 635-641. [CrossRef]

25. Ouf, S.A.; Gomha, S.M.; Ewies, M.M.; Sharawy, I.A.A. Synthesis, characterization and antifungal activity evaluation of some novel arylazothiazoles. J. Heterocycl. Chem. 2018, 55, 258-264. [CrossRef]

26. Shih, M.H.; Ke, F.Y. Syntheses and evaluation of antioxidant activity of sydnonyl substituted thiazolidinone and thiazoline derivatives. Bioorg. Med. Chem. 2004, 12, 4633-4643. [CrossRef] [PubMed]

27. Emam, D.R.; Alhajoj, A.M.; Elattar, K.M.; Kheder, N.A.; Fadda, A.A. Synthesis and Evaluation of Curcuminoid Analogues as Antioxidant and Antibacterial Agents. Molecules 2017, 22, 971. [CrossRef] [PubMed]

28. Mabkhot, Y.N.; Alatibi, F.; El-Sayed, N.N.; Al-Showiman, S.; Kheder, N.A.; Wadood, A.; Rauf, A.; Bawazeer, S.; Hadda, T. Antimicrobial Activity of Some Novel Armed Thiophene Derivatives and Petra/Osiris/Molinspiration (POM) Analyses. Molecules 2016, 21, 222. [CrossRef] [PubMed]

29. Mabkhot, Y.N.; Kheder, N.A.; Barakat, A.; Choudhary, M.I.; Yousuf, S.; Frey, W. Synthesis, antimicrobial, anti-cancer and molecular docking of two novel hitherto unreported thiophenes. RSC Adv. 2016, 6, 63724-63729. [CrossRef]

30. Gomha, S.M.; Kheder, N.A.; Abdelhamid, A.O.; Mabkhot, Y.N. One Pot Single Step Synthesis and Biological Evaluation of Some Novel Bis(1,3,4-thiadiazole) Derivatives as Potential Cytotoxic Agents. Molecules 2016, 21, 1532. [CrossRef] [PubMed]

31. Fun, H.-K.; Patil, P.S.; Dharmaprakash, S.M.; Chantraprommad, S.; Razak, I.A. (E)-3-(2,4-Dichlorophenyl) -1-(2-thienyl)prop-2-en-1-one. Acta Cryst. 2008, E64, o1814-o1815. [CrossRef] [PubMed]

32. Dieckmann, W.; Platz, L. Ueber eine neue bildungsweise von osotetrazonen. Ber. Dtsch. Chem. Ges. 1905, 38, 2986-2990. [CrossRef]

33. Abushamleh, A.S.; Al-Aqarbeh, M.M.; Day, V. Transition metal complexes of derivatized chiral dihydro1,2,4-triazin-6-ones. Template synthesis of nickel (II) tetraaza-(4N-M) complexes incorporating the triazinone moiety. Am. J. Appl. Sci. 2008, 5, 750-754. [CrossRef]

34. Marivel, S.; Braga, S.; Grepioni, F.; Lampronti, G.I. Mechanochemical preparation of adducts (co-crystals and molecular salts) of 1,4-diazabicyclo-[2.2.2]-octane with aromatic polycarboxylic acids. Cryst. Eng. Comm. 2010, 12, 2107-2112. [CrossRef]

35. Eweiss, N.F.; Abdelhamid, A.O. Synthesis of heterocycles. Part II. New routes to acetyl thiadiazolines and alkylazothiazoles. J. Heterocycl. Chem. 1980, 17, 1713-1717. [CrossRef]

36. Shawali, A.S.; Eweiss, N.F.; Hassaneen, H.M.; Al-gharib, M.S. Synthesis and rearrangement of ethyl aryloxyglyoxalate arylhydrazones. Bull. Chem. Soc. Jpn. 1975, 48, 365-366. [CrossRef]

37. Mosmann, T. Rapid colorimetric assay for cellular growth and survival: Application to proliferation and cytotoxicity assays. J. Immunol. Methods 1983, 65, 55-63. [CrossRef] 
38. Elaasser, M.M.; Abdel-Aziz, M.M.; El-Kassas, R.A. Antioxidant, antimicrobial, antiviral and antitumor activities of pyranone derivative obtained from aspergillus candidus. J. Microbiol. Biotech. Res. 2011, 1, 5-17.

39. Clinical and Laboratory Standards Institute. Performance Standards for Antimicrobial Susceptibility Testing; Twenty-Second Informational Supplement; CLSI Document M100-S22; CLSI: Wayne PA, USA, 2012; Available online: http://zums.ac.ir/files/health/pages/ill/azmayeshghah/clsi_2013.pdf (accessed on 13 November 2018).

40. Ghorab, M.M.; Alsaid, M.S.; El-Gaby, M.S.A.; Safwat, N.A.; Elaasser, M.M.; Soliman, A.M. Biological evaluation of some new $\mathrm{N}$-(2,6-dimethoxypyrimidinyl)thioureido benzenesulfonamide derivatives as potential antimicrobial and anticancer agents. Eur. J. Med. Chem. 2016, 124, 299-310. [CrossRef] [PubMed]

Sample Availability: Samples of the new synthesized pyrazolines are available from the authors.

(C) 2018 by the authors. Licensee MDPI, Basel, Switzerland. This article is an open access article distributed under the terms and conditions of the Creative Commons Attribution (CC BY) license (http:/ / creativecommons.org/licenses/by/4.0/). 